\title{
The Melting Temperature of a Thermally Reversible Gel. II. Ethylene-Vinyl Acetate Copolymer-Organic Solvent Gels
}

\author{
Akira TAKahashi \\ Department of Applied Chemistry, Nagoya University, Nagoya, Japan.
}

(Received June 28, 1972)

\begin{abstract}
Thermally reversible gels were formed from dilute solutions of ethylene - vinyl acetate copolymer (EVA) in dioxane, ethyl acetate, methyl ethyl ketone, and methyl isobutyl ketone. The power of solvents to form the gels were almost identical. The X-ray diffraction pattern of the EVA gels revealed that the gels were crystalline and that the crystallites, which serve as "cross-links", consisted of blocks of ethylene units in the copolymer. EVA copolymers, having ethylene mole fractions $\left(X_{\mathrm{A}}\right)$ from 0.64 to 0.84 , and ethyl acetate systems were studied in detail. The linear relationship between the reciprocal absolute melting temperature $\left(T_{\mathrm{m}} \mathrm{g}\right)$ and the logarithm of $v_{2} x$ was found for each EVA, where $v_{2}$ is the volume fraction of polymer and $x$ the degree of polymerization. The results were analysed using the theoretical expression given in the previous paper. The analysis leads to the conclusion that the ethylene sequence length in the crystallites, i.e., the block length of ethylene unit in the copolymer, is 10 irrespective of $X_{\mathrm{A}}$. It was also shown that the conclusion is in accordance with the block length estimated by Wu from the 220-MHz NMR spectra of EVA-copolymer solutions.
\end{abstract}

KEY WORDS Gel / Melting Temperature of Gel / Ethylene-Vinyl Acetate Copolymer / Thermally Reversible Gel / Crystallite Length /

In the past decade, the revived research on the thermally reversible gels has been carried out. The extensive studies of thermally reversible gels formed from various types of synthetic polymers have been reported by Haas and his coworkers. ${ }^{1}$ Especially, they have succeeded in preparing the "synthetic thermally reversible gel systems" such as poly(acrylglycinamide) in water, and concluded that in the above system, a "cross-link" consists of only one group from each of the two polymeric chains.

However, the nature and the structure of the thermally reversible cross-links are generally very complex. It is conceivable almost all types of bonds and interactions, such as hydrogen bonds, hydrophobic bonds, electrostatic interactions as well as dipole interactions, may be candidates for the "cross-links". ${ }^{1}$ The crystallites consisting of crystalline sequences in polymer chains can also serve as the "cross-links". Therefore, it is of primary importance to clarify the nature and the structure of the cross-links. Moreover, the polymer-solvent interaction, especially the free energy of dilution, is also an important factor for thermally reversible gelation. In the previous paper of this series, ${ }^{2}$ it has been shown that a more detailed analysis of the above problems is possible, by using a poly(vinyl chloride)-organic solvent gel. Taking into account the copolymeric character which is conductive to gel formation, and then considering both the conditions at the gel point ${ }^{3}$ as well as the free energy of formation of crystallites which serve as the "cross-links", the following equation has been derived ${ }^{2}$

$$
\begin{aligned}
\frac{1}{T_{\mathrm{m}} \mathrm{g}}= & \frac{\zeta}{\left(\Delta h_{\mathrm{u}} \zeta+B^{\prime} V_{\mathrm{A}} \zeta-2 \sigma_{\mathrm{ec}}\right)} \\
& \times\left(\frac{\Delta h_{\mathrm{u}}}{T_{\mathrm{m}}^{\circ}}+\frac{R V_{\mathrm{A}}}{V_{1}}-R \ln X_{\mathrm{A}}\right) \\
& -\frac{R}{\left(\Delta h_{\mathrm{u}} \zeta+B^{\prime} V_{\mathrm{A}} \zeta-2 \sigma_{\mathrm{ec}}\right)} \ln v_{2} x
\end{aligned}
$$

In the above, $T_{\mathrm{m}} \mathrm{g}$ is the melting temperature of gel, $v_{2}$ is the volume fraction of the copolymer in the gel, $x$ is the number of the repeating unit in the polymer, $\zeta$ is the number of crystallized unit $A$ which has entered into the crystal- 


\section{A. TAKahashi}

lite as a sequence, $\Delta h_{\mathrm{u}}$ is the heat of fusion per repeating unit, $T_{\mathrm{m}}{ }^{\circ}$ is the equilibrium melting temperature, $X_{\mathrm{A}}$ is the mole fraction of crystallizable unit $A$ in the polymer, $B^{\prime}$ is the interaction energy density characteristic of the "polymer-solvent" pair (cf. seq.), $\sigma_{\text {ec }}$ is the end interfacial free energy per crystalline sequence, and $V_{\mathrm{A}}$ and $V_{1}$ are the molar volumes of the unit $A$ and solvent, respectively.

The gel melting temperature of the poly(vinyl chloride) gels were analysed by using eq 1 , and it has been shown that it well represents the $T_{\mathrm{m}}{ }^{\mathrm{g}}$ of the gels as a function of $v_{2}, x$, and $B^{\prime}$. However, the conclusion has only been reached when $X_{\mathrm{A}}$ is constant, since poly(vinyl chloride) samples have been used which have constant $X_{\mathrm{A}}$, different $x$, and almost identical syndiotacticity. Therefore, further work is necessary to confirm that eq 1 is still valid even if $X_{\mathrm{A}}$ is varied.

Ethylene-vinyl acetate copolymer (EVA) is suitable for this purpose, since EVA copolymers which differ in $X_{\mathrm{A}}$ are easily available now. The purpose of this paper is, (1) to confirm that EVA copolymer-diluent systems really form the thermally reversible gels, and (2) to show that the theoretically derived eq 1 is also applicable for this copolymer gel.

\section{EXPERIMENTAL}

\section{Materials}

Ethylene-vinyl acetate copolymers (EVA), which range in mole fraction of ethylene content in the copolymer from 0.64 to 0.84 , were dissolved in benzene and precipitated by methanol, and finally dried in vacuo at $45^{\circ} \mathrm{C}$ for 3 days. Dioxane, ethyl acetate (EAc), methyl ethyl ketone (MEK), and methyl isobutyl ketone (MIBK) were purified by distillation before use.

\section{Characterization of Polymers}

The mole fractions of ethylene unit $\left(X_{\mathrm{A}}\right)$ in the copolymers were determined by elementary analysis. Their number-average molecular weights were determined by osmometry using a Mechrolab High Speed Membrane Osmometer fitted with a $0-8$ membrane. Ethyl acetate was employed as solvent and the measurements were carried out at $30^{\circ} \mathrm{C}$. To ensure the complete
Table I. Molecular weights and mole fractions of the ethylene unit $\left(X_{\mathrm{A}}\right)$ in EVA copolymers

\begin{tabular}{ccrc}
\hline Sample & $M_{\mathrm{n}} \times 10^{-4}$ & \multicolumn{1}{c}{$x$} & $X_{\mathrm{A}}$ \\
\hline EVA-84 & 3.7 & 929 & 0.84 \\
EVA-75 & $6.4_{4}$ & 1380 & 0.75 \\
EVA-71 & 6.9 & 1390 & 0.71 \\
EVA-64 & $9.4_{6}$ & 1740 & 0.64 \\
\hline
\end{tabular}

dissolution of the copolymers in EAc and to avoid molecular association, all solutions were warmed to $45^{\circ} \mathrm{C}$, kept for several hours at this temperature prior to carrying out the osmotic pressure measurements and never cooled below $30^{\circ} \mathrm{C}$.

The details of the samples are given in Table I.

\section{The Gel Melting Temperature}

The method of the preparation of gels and the measurements of their melting temperatures were described previously. ${ }^{2}$ For EVA-84, 75 and and 71 , the gelation was carried out at $0^{\circ} \mathrm{C}$ for $20 \mathrm{hr}$, whereas for EVA- $64,-30^{\circ} \mathrm{C}$ was employed for gelation since gelation at $0^{\circ} \mathrm{C}$ resulted in very weak gels.

\section{$X$-ray Diffraction of the Gel}

Molten gels were injected into a fine silica capillary (1-mm inner diameter) with a hypodermic needle and gelation was conducted at $0^{\circ} \mathrm{C}$ for $48 \mathrm{hr}$ in the capillary. The capillary was mounted on a Toshiba X-ray diffraction camera and the X-ray diffraction pattern was obtained by using $\mathrm{Cu}-\mathrm{K}_{\alpha}$ point beam.

\section{RESULTS AND DISCUSSION}

\section{Effect of Solvent on $T_{\mathrm{m}} \mathrm{g}$}

In order to find out whether the EVA copolymer-organic solvent systems turn into gels when the temperature is changed, EVA-84 was selected, and its dioxane, EAc, MEK, and MIBK solutions were prepared by warming. The resultant solutions were introduced into the gel melting temperature apparatus and stored for $20 \mathrm{hr}$ at $0^{\circ} \mathrm{C}$. All of them turned into transparent gels. Their melting temperatures were measured by the procedure described previously, ${ }^{2}$ and examples of the measurements 
of $T_{\mathrm{m}} \mathrm{g}$ are shown in Figure 1.

In Figure 2 the reciprocals of $T_{\mathrm{m}} \mathrm{g}$ are plotted against $\ln v_{2} x$. Although the experimental points for different solvents are scattered about the straight line drawn in the figure, it is suggested that the influence of the solvents on

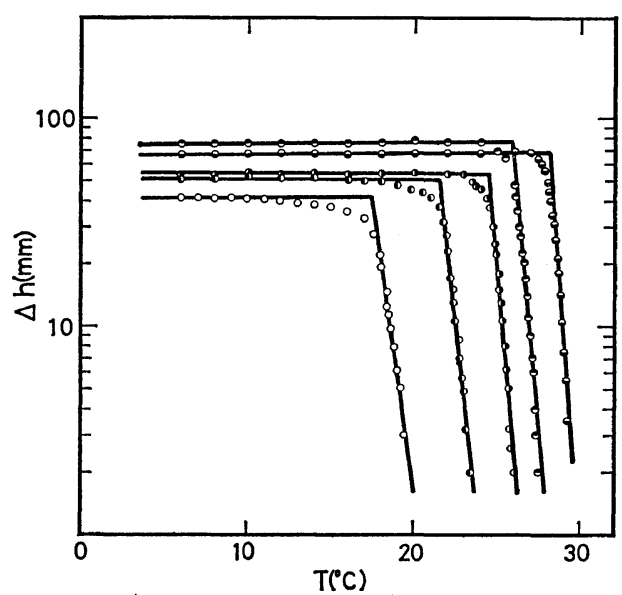

Figure 1. Examples for determination of $T_{\mathrm{m}} \mathrm{g}$ : sample EVA-71 in EAc. Volume fractions of polymer: $\bigcirc, 0.0365$; $D, 0.0452 ; \bigcirc, 0.0538$; 0.0622; $\odot, 0,0704$.

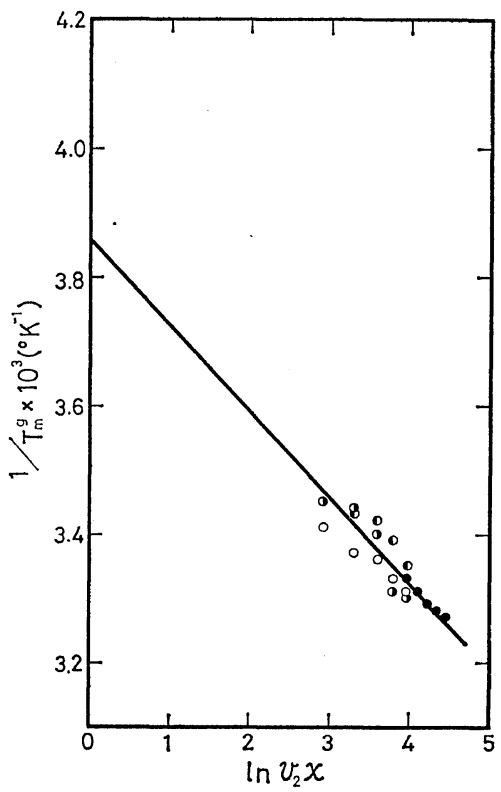

Figure 2. Plots of $1 / T_{\mathrm{m}^{\mathrm{g}}} v s . \ln v_{2} x$ for four EVA84 gels: $\bigcirc$, EAc; $\bigcirc$, dioxane; $($, MEK; $D$, MIBK.
$T_{\mathrm{m}} \mathrm{g}$ is almost the same. This fact is easily understood if one compares the solubility parameters $(\delta)$ of the four solvents EAc, MEK, MIBK, and dioxane. Their values for $\delta$ are 9.0, 9.3, 9.5, and 9.8 , respectively, ${ }^{4}$ i.e., almost the same magnitude. EAc has therefore been used as the only solvent for the following detailed studies of the EVA gels.

\section{$X$-ray Diffraction}

In order to clarify the nature of the "crosslinks" in the EVA gels, the X-ray diffraction pattern of the gels was examined. Typical Xray diffraction patterns of EVA-71-EAc gel are shown in Figure 3a. The polymer concentration in the gel was $12 \mathrm{~g} / 100 \mathrm{ml}$. Similar diffraction patterns have also been obtained for the gels that were formed from the other EVA copolymers. In Figure 3a, two diffraction rings are

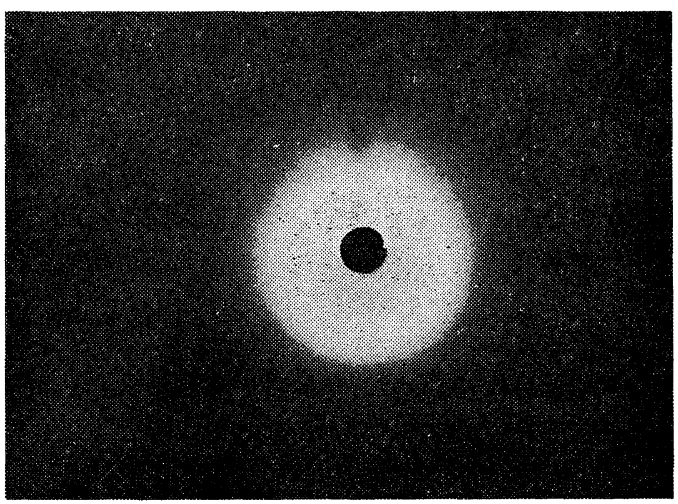

(a)

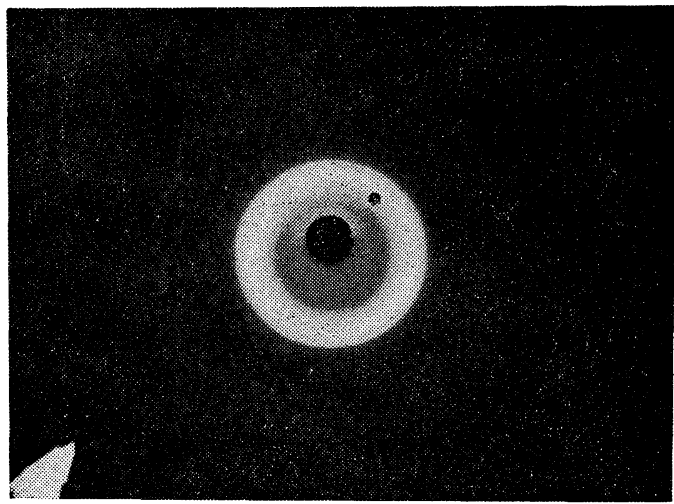

(b)

Figure 3. X-ray diffraction patterns of (a) EVA71 -EAc gel $(C=12 \mathrm{~g} / 100 \mathrm{~m} l)$ and (b) EVA copolymer film crystallized at $100^{\circ} \mathrm{C}$ for $240 \mathrm{hr}$. 


\section{A. TAKahashi}

observed; the inner ring is intense but diffuse and wide, whereas the outer ring has very weak diffraction intensity but is fairly sharp.

For comparison, the X-ray diffraction pattern of EVA-copolymer film (ethylene mole fraction, 0.973 ), crystallized at $100^{\circ} \mathrm{C}$ for $240 \mathrm{hr}$, is shown in Figure 3b. In the latter, by comparison with the result of Bunn, ${ }^{5}$ two intense inner diffraction rings, which correspond to $2 \theta=21^{\circ} 38^{\prime}$ and $2 \theta=24^{\circ} 09^{\prime}$ or $d=4.10_{1} \AA$ and $d=3.68_{9} \AA$ are identified as 110 and 200 reflections from the polyethylene crystal, respectively. The other outer rings having very weak intensities, could also be identified by comparison with Bunn's data, and their $h k l$ are $020,011,211$, and $(320,410)$, respectively. The comparison between Figures $3 a$ and $3 b$ reveals that two intense 110 and 200 reflections, which have been observed for the bulk-crystallized EVA film, were merged into one wide ring in the case of the EVA-EAc gel. The other outer rings in Figure $3 b$ diffused into the background except the outermost ring corresponding to 320 and 410 reflections.

Since conventional poly(vinyl acetate) is known to be a noncrystalline polymer and the transparent EVA gels have been proved to be crystalline from $\mathrm{X}$-ray observation, one may conclude that the crystallites may consist of the ethylene blocks in the EVA chains. Furthermore, judging from the diffuseness of the ring, the crystallites may be supposed to be very small in size, i.e., the block length of the ethylene unit in the EVA is short, but sufficiently long to form the crystallites which serve as "cross-links".

\section{Effect of Ethylene Mole Fraction on $T_{\mathrm{m}} \mathrm{g}$}

In Figure 4 , the plots of $1 / T_{\mathrm{m}} \mathrm{g}$ against $\ln v_{2} x$ are shown for four EVA-copolymer gels. Apparently the linear relationship between $1 / T_{\mathrm{m}} \mathrm{g}$ and $\ln v_{2} x$ also holds in this case, as originally pointed out by Eldridge are Ferry. ${ }^{6}$ Since the EVA copolymers differ in ethylene mole fraction, the results indicate the effect of $X_{\mathrm{A}}$ on $T_{\mathrm{m}}{ }^{\mathrm{g}}$.

In order to analyse the results according to eq 1 , one needs to know $B^{\prime}$ besides $T_{\mathrm{m}}{ }^{\circ}$ and $\Delta h_{\mathrm{u}}$. Flory ${ }^{7}$ has pointed out that the "constant" $B^{\prime}$ will depend on the composition of the "solvent medium" which consists of the diluent and the noncrystallized units in the copolymer. Thus, the definition of $B^{\prime}$ in this case is somewhat

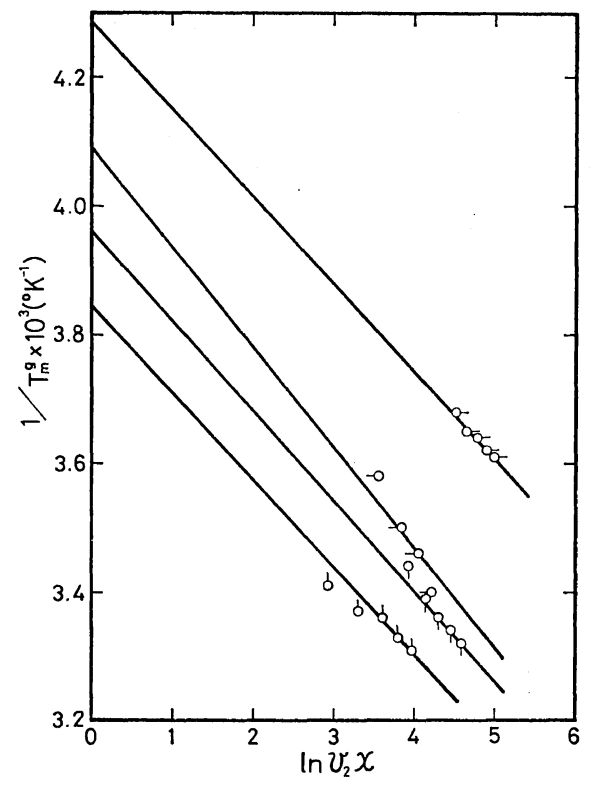

Figure 4. Plots of $1 / T_{\mathrm{m}^{\mathrm{g}}}$ against $\ln v_{2} x$ for four different EVA gels. $X_{\mathrm{A}}$ : $\bigcirc, 0.84 ;-\bigcirc, 0.75$; ○, 0.71; $\bigcirc^{-}, 0.64$.

different from the usual definition of the cohesive energy density of homopolymer-diluent system. Since the experimental determination of $B^{\prime}$ is difficult, it was assumed that an approximate evaluation of $B^{\prime}$ is possible from the observed second virial coefficient $\left(A_{2}\right)$ by making use of the relationship for polymer solution ${ }^{8}$

$$
A_{2}=\frac{\bar{v}^{2}}{V_{1}}\left(\frac{1}{2}-\chi\right)
$$

and

$$
\chi=\frac{B^{\prime} V_{1}}{R T}
$$

where $\bar{v}$ is the partial specific volume of the polymer and $\chi$ is the interaction parameter. In the subsequent analysis, however, it will become apparent that the approximate evaluation of $B^{\prime}$ does not result any serious error in the estimation of $\zeta$ and $\sigma_{\mathrm{ec}}$. The result of osmotic pressure measurements are shown in Figure 5, and the values of $A_{2}, B^{\prime}$, and $\chi$ are summarized in Table II.

Making use of the authors' previously reported procedure ${ }^{2}$ and adopting the values of $T_{\mathrm{m}}{ }^{\circ}=145.5^{\circ} \mathrm{C},{ }^{9} \quad \Delta h_{\mathrm{u}}=1920 \mathrm{cal} / \mathrm{mol}$ of ethylene 
Table II. The second virial coefficient $A_{2}, B^{\prime}, \chi$, and the estimation of $\zeta$ and $\sigma_{\mathrm{ec}}$ for the EVA copolymers-ethyl acetate systems

\begin{tabular}{|c|c|c|c|c|c|c|c|c|}
\hline \multirow[b]{2}{*}{ Sample } & \multirow[b]{2}{*}{$X_{\mathrm{A}}$} & \multirow[b]{2}{*}{$\begin{array}{l}A_{2} \times 10^{4}, \\
\mathrm{cc} \mathrm{mol} / \mathrm{g}^{2}\end{array}$} & \multirow{2}{*}{$\begin{array}{c}B^{\prime} \\
\mathrm{cal} / \mathrm{cc}\end{array}$} & \multirow[b]{2}{*}{$\chi$} & \multicolumn{2}{|c|}{ Obsd } & \multirow[b]{2}{*}{$\zeta^{\mathrm{a}}$} & \multirow[b]{2}{*}{$\begin{array}{c}\sigma_{\mathrm{ec}} \\
\mathrm{cal} / \mathrm{mol}\end{array}$} \\
\hline & & & & & $\begin{array}{c}\text { Intercept } \\
\times 10^{3},{ }^{\circ} \mathrm{K}^{-1}\end{array}$ & $\begin{array}{c}\text { Slope } \\
\times 10^{4},{ }^{\circ} \mathbf{K}^{-1}\end{array}$ & & \\
\hline EVA-84 & 0.84 & 4.00 & 2.83 & 0.463 & 3.84 & 1.35 & 10.4 & 2950 \\
\hline EVA-75 & 0.75 & 3.89 & 2.81 & 0.461 & 4.09 & 1.55 & 9.4 & 2800 \\
\hline EVA-71 & 0.71 & 6.10 & 2.66 & 0.437 & 3.96 & 1.40 & 9.8 & 2600 \\
\hline \multirow[t]{2}{*}{ EVA-64 } & 0.64 & 8.44 & 2.49 & 0.408 & 4.28 & 1.35 & 10.5 & 3000 \\
\hline & & & & & & & av 10.0 & 2837 \\
\hline
\end{tabular}

${ }^{a} \zeta$ is expressed in number of ethylene units.

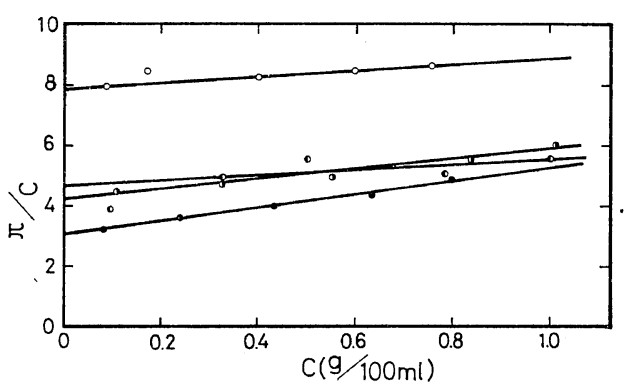

Figure 5. The osmotic pressures of EVA copolymers in ethyl acetate at $30^{\circ} \mathrm{C}$ : $\bigcirc$, EVA-84; $\mathbf{D}$, $75 ;$ (1) 71; , 64 .

unit, ${ }^{10}$ and the values of both $B^{\prime}$ and $X_{A}$ for each EVA copolymer, one may determine $\zeta$ and $\sigma_{\text {ec }}$ from the observed intercept and the slope, respectively. The results are shown in Table II. It is noticeable that the values of both $\zeta$ and $\sigma_{\mathrm{ec}}$ are almost constant for the four EVA-copolymer gels.

The fact that $\zeta=10$ has been obtained indicates the existence of blocks of 10 or more ethylene units in the copolymer chain. This conclusion is further substantiated by comparison with the result reported by $\mathrm{Wu} .^{11} \mathrm{Wu}$ has studied 220$\mathrm{MHz}$ proton nuclear magnetic resonance spectra of EVA copolymers in aromatic solvents. For EVA copolymers containing ethylene units more than $X_{\mathrm{A}}=0.625$, he observed that the methylene protons of the alkane-like sequences give rise two resonance peaks, and these two peaks have been assigned to the two types of intramolecular structure, the one is "polymeric" and the other is "monomeric". Following the experimental observations of Liu and Ullman ${ }^{12}$ on the polyethylene oligomers, the origin of the "polymeric" peak has been attributed to the chain folding of the long ethylene sequences containing eight or more ethylene units. Moreover, $\mathrm{Wu}$ has compared the observed fractions of ethylene units in blocks of eight or more ethylene units with the calculated. For statistically random EVA copolymerization, ${ }^{11}$ the fraction $G(n)$ of ethylene in blocks of $n$ or more units of ethylene can be calculated by the equation

$$
G(n)=X_{\mathrm{A}}^{n}\left[1+(n-1)\left(1-X_{\mathrm{A}}\right)\right]
$$

It has been observed that the observed $G(8)$ is always smaller than the calculated $G(8)$. For the EVA copolymers which have almost the same $X_{\mathrm{A}}$ as used here, the following values of $G(8)_{\text {calc }}$ and $G(8)_{\text {obsd }}$ were reported: $\quad G(8)_{\text {calc }}=0.084$ and $G(8)_{\text {obsd }}=0$ for $X_{\mathrm{A}}=0.625 ; G(8)_{\mathrm{calc}}=0.697$ and $G(8)_{\text {obsd }}=0.34$ for $X_{\mathrm{A}}=0.867$. Thus, there are substantial fractions of ethylene blocks $n \geq 8$ which can form the crystallites in the gel. The reason why the gelation at $-30^{\circ} \mathrm{C}$ was necessary for the sample EVA-64 may be explained by the fact that $G(8)_{\mathrm{obsd}} \simeq 0$ for $X_{\mathrm{A}}=0.625$. Since the fractions of ethylene sequences containing eight or more ethylene units in EVA-64 is very small, so that very large undercooling was necessary in comparison with the gelation of the other EVA copolymers which have higher ethylene contents than EVA-64.

Since the physical basis of the NMR spectroscopy and the analysis of melting temperature of the gel are quite different, the fact that the same conclusion has been reached concerning the size of the ethylene blocks supports the validity of the present analysis of the gel melting temperature. 


\section{A. TAKahashi}

It is also interesting to note that the average value of $\sigma_{\mathrm{ec}}=2840 \mathrm{cal} / \mathrm{mol}$ is very close to the interfacial free energy of polyethylene single crystals deduced from their dissolution temperature in xylene. ${ }^{13}$ However, considering the difference between the interfacial structure of the single crystal (where chain folding occurs) and that of the crystallite in the gel (which may be considered to resemble the fringed-micelle-type crystallite), no conclusion can be reached regarding the interfacial structure from the value of $\sigma_{\mathrm{ec}}$.

Eq 1 may be rearranged as

$$
\begin{aligned}
& {\left[\frac{1}{T_{\mathrm{m}} \mathrm{g}}+\frac{\zeta R \ln X_{\mathrm{A}}}{\left(\Delta h_{\mathrm{u}} \zeta+B^{\prime} V_{\mathrm{A}} \zeta-2 \sigma_{\mathrm{ec}}\right)}\right]} \\
& =\frac{\frac{\Delta h_{\mathrm{u}}}{T_{\mathrm{m}}{ }^{\circ}}+R\left(\frac{V_{\mathrm{A}}}{V_{1}}\right)}{\left(\Delta h_{\mathrm{u}} \zeta+B^{\prime} V_{\mathrm{A}} \zeta-2 \sigma_{\mathrm{ec}}\right)} \\
& \quad-\frac{R}{\left(\Delta h_{\mathrm{u}} \zeta+B^{\prime} V_{\mathrm{A}} \zeta-2 \sigma_{\mathrm{ec}}\right)} \ln v_{2} x
\end{aligned}
$$

According to eq 4 , when $\zeta$ and $\sigma_{\mathrm{ec}}$ are constant independent of $X_{A}$, and furthermore, $B^{\prime} V_{A} \zeta$ $\ll\left(\Delta h_{\mathrm{u}} \zeta-2 \sigma_{\mathrm{ec}}\right)$, then it is expected that the plots of the left hand side of eq 4 vs. $\ln v_{2} x$ will be reduced almost to a straight line. In Figure 6 , these plots are shown; where the left hand side of eq 4 was calculated from the values of

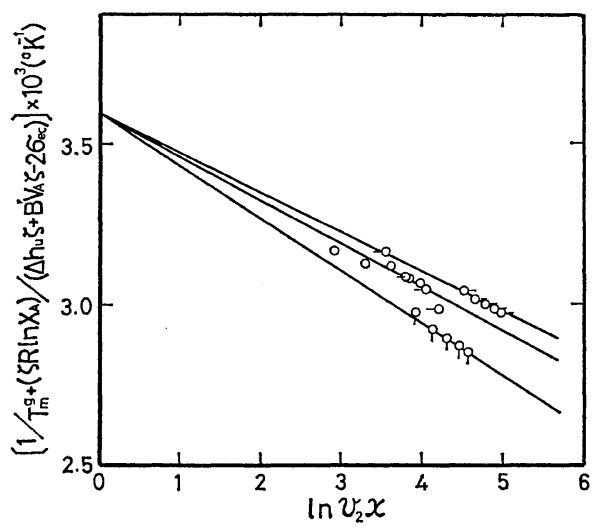

Figure 6. Plots of $\left[\left(1 / T_{\mathrm{m}}^{\mathrm{g}}\right)+\left(\zeta R \ln X_{\mathrm{A}}\right) /\left(\Delta h_{\mathrm{u}} \zeta+\right.\right.$ $\left.\left.B^{\prime} V_{\mathrm{A}} \zeta-2 \sigma_{\mathrm{ec}}\right)\right] v s . \ln v_{2} x$. The symbols for $X_{\mathrm{A}}$ are the same as in Figure 4.
$X_{\mathrm{A}}, \zeta, B^{\prime}$, and $\sigma_{e \mathrm{ec}}$ in Table II and also from the observed $T_{\mathrm{m}}{ }^{\mathrm{g}}$. Although the lines in the figure have slightly different slopes, a common intercept is obtained and one may consider that the expectation is almost fulfilled.

In conclusion, it has been confimed that eq 1 is quite satisfactory for analysing the melting temperatures of the copolymer gels.

Acknowledgment. Special thanks are due to Mr. Noboru Ueno for his assistance in the measurements of the gel melting temperature, and to Professor Ikumi Kagawa for his encouragement throughout this research.

\section{REFERENCES}

1. H. C. Haas, M. J. Manning, and S. A. Hollander, "Analytical Calorimetry," Vol. II, Plenum Press, New York, N. Y., 1970, p 211. Therein, the references of their studies on thermally reversible gels are collected.

2. A. Takahashi, T. Nakamura, and I. Kagawa, Polymer J., 3, 207 (1972).

3. P. J. Flory, "Principles of Polymer Chemistry," Cornell University Press, Ithaca, N. Y., 1953, Chapter IX.

4. J. H. Hildebrand and R. L. Scott, "Solubility of Non-electrolyte," 3rd ed., Reinhold Publishing Co., 1950.

5. C. W. Bunn, Trans. Faraday Soc., 35, 482 (1939).

6. J. E. Eldridge and J. D. Ferry, J. Phys. Chem., 58, 992 (1954).

7. P. J. Flory, J. Chem. Phys., 17, 223 (1949).

8. P. J. Flory, "Principles of Polymer Chemistry," Cornell University Press, Ithaca, N. Y., 1953, Chapter XII.

9. P. J. Flory and A. Vrij, J. Amer. Chem. Soc., 85, 3548 (1963).

10. F. A. Quinn, Jr., and L. Mandelkern, ibid., 80, 3178 (1958).

11. T. -K. Wu, Macromolecules, 2, 520 (1969).

12. K.-J. Liu, J. Polym. Sci., Part A-2, 5, 1209 (1967); K. -J. Liu and R. Ullman, ibid., Part $A-2,6,451$ (1968).

13. J. F. Jackson and L. Mandelkern, Macromolecules, 1, 546 (1968). 